\title{
Convergence of iterates of linear operators and the Kelisky-Rivlin type theorems
}

by

\author{
JACEK JACHYMSKI (Łódź)
}

\begin{abstract}
Let $X$ be a Banach space and $T \in L(X)$, the space of all bounded linear operators on $X$. We give a list of necessary and sufficient conditions for the uniform stability of $T$, that is, for the convergence of the sequence $\left(T^{n}\right)_{n \in \mathbb{N}}$ of iterates of $T$ in the uniform topology of $L(X)$. In particular, $T$ is uniformly stable iff for some $p \in \mathbb{N}$, the restriction of the $p$ th iterate of $T$ to the range of $I-T$ is a Banach contraction. Our proof is elementary: It uses simple facts from linear algebra, and the Banach Contraction Principle. As a consequence, we obtain a theorem on the uniform convergence of iterates of some positive linear operators on $C(\Omega)$, which generalizes and subsumes many earlier results including, the Kelisky-Rivlin theorem for univariate Bernstein operators, and its extensions for multivariate Bernstein polynomials over simplices. As another application, we also get a new theorem in this setting giving a formula for the limit of iterates of the tensor product Bernstein operators.
\end{abstract}

1. Introduction. Let $L(X)$ be the space of all bounded linear operators on a Banach space $X$. Given $T \in L(X)$ and $n \in \mathbb{N}$, the set of all positive integers, we denote by $T^{n}$ the $n$th iterate of $T$. Set

$$
\begin{aligned}
& D_{T^{\infty}}:=\left\{x \in X:\|\cdot\|-\lim _{n \rightarrow \infty} T^{n} x \text { exists }\right\}, \\
& T^{\infty} x:=\lim _{n \rightarrow \infty} T^{n} x \quad \text { for } x \in D_{T^{\infty}} .
\end{aligned}
$$

Clearly, $D_{T^{\infty}}$ is always a linear $T$-invariant subspace of $X$, and $T^{\infty}$ is a linear operator. If $D_{T^{\infty}}=X$, which means $\left(T^{n}\right)_{n \in \mathbb{N}}$ is convergent to $T^{\infty}$ in the strong topology of $L(X)$, then, by the Banach-Steinhaus theorem, the operator $T^{\infty}$ is bounded. In this case $T$ is also called strongly stable (see, e.g., [Em07, p. 3]). There are a number of results in the literature (see, e.g., [AA96], [AR03], [CF86], [CF93], [GP05], [KR67], [OT02], [Ru04], [We97]) showing that some particular linear operators are strongly stable,

2010 Mathematics Subject Classification: Primary 47A35, 47B38; Secondary 47A10, $47 \mathrm{~B} 65$.

Key words and phrases: iterates of linear operators, uniformly stable operator, spectral radius, positive linear operators, univariate and multivariate Bernstein operators, $q$-Bernstein operators, tensor product Bernstein operators. 
and giving a formula for the operator $T^{\infty}$. For example, the following theorem was proved in [KR67].

Theorem 1 (Kelisky-Rivlin). For $m \in \mathbb{N}$ and $f \in C[0,1]$, let $B_{m} f$ be the Bernstein polynomial of degree $m$ associated with $f$, i.e.,

$$
\left(B_{m} f\right)(t):=\sum_{k=0}^{m} f\left(\frac{k}{m}\right)\left(\begin{array}{c}
m \\
k
\end{array}\right) t^{k}(1-t)^{m-k} \quad \text { for } t \in[0,1] .
$$

Then the sequence $\left(B_{m}^{n}\right)_{n \in \mathbb{N}}$ strongly converges to the operator $B^{\infty}$ (independent of $m$ ) given by the following formula:

$$
\left(B^{\infty} f\right)(t)=f(0)+(f(1)-f(0)) t \quad \text { for } f \in C[0,1] \text { and } t \in[0,1] .
$$

The original proof of Theorem 1 is rather involved; in particular, it uses the Stirling numbers of the second kind. Subsequently, Chang and Feng [CF86] extended Theorem 1 to the case of bivariate Bernstein polynomials over a triangle (cf. also [AA96]). Another proof of their result was given by Wenz [We97], but his argument is also complicated and it requires some hard calculations. As pointed out in [We97], these ideas could be adapted to the general setting of more than two variables, but the proofs would become yet more technical.

Our purpose here is twofold. First, in Section 2, we give necessary and sufficient conditions for the convergence of $\left(T^{n}\right)_{n \in \mathbb{N}}$ in the uniform topology of $L(X)$ (then $T$ is also called uniformly stable; cf. [Em07, p. 21]) for any linear operator $T$ on an arbitrary Banach space $X$. Our proof is elementary: It uses some simple facts from linear algebra, and the Banach Contraction Principle. In fact, it was inspired by Rus' [Ru04] argument used in his elegant proof of the Kelisky-Rivlin theorem, and subsequently extended to the case of an arbitrary Banach space in our paper [Ja07]. In particular, Theorem 5 generalizes [Ja07, Theorem 4.1] in which a sufficient condition for the uniform convergence of $\left(T^{n}\right)_{n \in \mathbb{N}}$ was given. However, after completing a preliminary version of this paper, we found that we had partially rediscovered the following spectral criterion of Koliha [Ko73, Theorem 4 and Corollary 5]. (For the notions of spectral theory used below, see, e.g., [DS57]; we denote by cl $A$ the closure of a set $A \subseteq X$ with respect to the norm topology.)

Theorem 2 (Koliha). For an operator $T \in L(X)$, the following statements are equivalent:

(i) $\left(T^{n}\right)_{n \in \mathbb{N}}$ is uniformly convergent in $L(X)$;

(ii) the spectrum $\sigma(T)$ is contained in $D \cup\{1\}$ (where $D$ denotes the open unit disc), and the point 1 is a simple pole of the resolvent $R_{\lambda}(T)$ whenever $1 \in \sigma(T)$;

(iii) the spectrum of the restriction $T_{1}$ of $T$ to the subspace $\operatorname{cl}(I-T)(X)$ is contained in $D$. 
Clearly, (iii) means that $r\left(T_{1}\right)$, the spectral radius of $T_{1}$, is less than 1 , and this condition is equivalent to (vi) of Theorem 5 below. However, we give a complete proof of Theorem 5 since our methods are different; in particular, we do not refer to any properties of points of the spectrum, and we add some new equivalent conditions. After all, it seems Koliha's result is not well known. For example, it is not cited in the recent monograph [Em07] though the following theorem of Räbiger [Rä93, Lemma 2.5] is given there (cf. [Em07, p. 21]). (We denote by $\sigma_{\pi}(T)$ the peripheral spectrum of $T ; T$ is called uniformly mean ergodic if the sequence $\left(\sum_{k=0}^{n-1} T^{k} / n\right)_{n \in \mathbb{N}}$ of Cesàro means uniformly converges in $L(X)$.)

Theorem 3 (Räbiger). Let $T \in L(X)$. Then $\left(T^{n}\right)_{n \in \mathbb{N}}$ is uniformly convergent if and only if $T$ is uniformly mean ergodic and $\sigma_{\pi}(T) \subseteq\{1\}$.

Hence by the uniform ergodic theorem of Lin [Li74], we get the following equivalence: $\left(T^{n}\right)_{n \in \mathbb{N}}$ uniformly converges iff $\left\|T^{n}\right\| / n \rightarrow 0$, the subspace $(I-T)(X)$ is closed and $\sigma_{\pi}(T) \subseteq\{1\}$. Independently, Mbekhta and Zemánek [MZ93, Cor. 3] proved the following result.

Theorem 4 (Mbekhta-Zemánek). Let $T \in L(X)$. The following statements are equivalent:

(i) $\left(T^{n}\right)_{n \in \mathbb{N}}$ is uniformly convergent in $L(X)$;

(ii) $\left\|T^{n}\right\| / n \rightarrow 0,(I-T)^{m}(X)$ is closed for some $m \in \mathbb{N}$ and $\sigma(T) \cap \Gamma$ $\subseteq\{1\}$, where $\Gamma$ denotes the unit circle;

(iii) $\left\|T^{n}-T^{n+1}\right\| \rightarrow 0$ and $(I-T)^{m}(X)$ is closed for some $m \in \mathbb{N}$.

However, it seems that if an operator $T$ is given explicitly, then it may be inconvenient to use the above results in order to deduce the uniform stability of $T$. Thus, our second purpose is to demonstrate that in that case Theorem 5 can be useful. In particular, as a consequence, we obtain Theorem 6 (cf. Section 3) on the uniform convergence of iterates of some positive linear operators on $C(\Omega)$, the space of all continuous functions on a compact topological space $\Omega$. Here we also give a formula for the operator $T^{\infty}$. Theorem 6 generalizes and subsumes many results of other authors. In particular, it easily yields Theorem 1, the above mentioned theorem of Wenz [We97, Theorem 7] for bivariate Bernstein polynomials over a triangle, a result of Oruç and Tuncer [OT02, Theorem 3.1] for the so-called $q$-Bernstein operators introduced by Phillips [Ph97], and a theorem of Gonska and Piţul [GP05, Theorem 3.2] for generalized Bernstein operators defined recently by King [Ki03]. Moreover, a novelty here is Theorem 11 on the uniform convergence of iterates of the Bernstein polynomials over the square, together with a formula on the limit of iterates, which may easily be extended to the case of polynomials over the $N$-cube. 
Finally, let us note that a spectral criterion for the uniform convergence of $\left(T^{n}\right)_{n \in \mathbb{N}}$ was also obtained by Li [Li86] (the paper in Chinese). (His result is also mentioned in [LZ94].) However, at least the version of Li's theorem presented in the review [Ge86] seems to be incorrect. In particular, the author claims that if $\left(T^{n}\right)_{n \in \mathbb{N}}$ uniformly converges, then either $r(T)<1$ (in this case $\left.T^{\infty}=0\right)$ or $\sup \{|\lambda|: \lambda \neq 1, \lambda \in \sigma(T)\}=1$. By compactness of $\sigma(T)$, the latter condition implies the existence of $\lambda_{0} \in \sigma(T)$ and $\left(\lambda_{n}\right)_{n \in \mathbb{N}}$ such that $\lambda_{n} \neq 1, \lambda_{n} \in \sigma(T), \lambda_{n} \rightarrow \lambda_{0}$ and $\left|\lambda_{0}\right|=1$. Then, by Theorem $2, \lambda_{0}=1$, which yields a contradiction since the point 1 , being a simple pole of $R_{\lambda}(T)$, is an isolated point of $\sigma(T)$.

We denote by $\operatorname{ker} T$ the null space of $T$, and by Fix $T$ the subspace of all fixed points of $T$. Throughout the paper, the notation $X=Y \oplus Z$ means $X$ is a topological direct sum of linear subspaces $Y$ and $Z$; in particular, $Y$ and $Z$ are then closed.

2. Uniform stability: Equivalent conditions. We start with the following auxiliary result.

Lemma 1. Let $X$ be a linear space, $X_{0}$ be a linear subspace of $X$, and $T$ be a linear operator on $X$. The following statements are equivalent:

(i) $(I-T)(X) \subseteq X_{0}$;

(ii) $T\left(x+X_{0}\right) \subseteq x+X_{0}$ for any $x \in X$, i.e., every coset of the quotient space $X / X_{0}$ is $T$-invariant.

In particular, $(I-T)(X)$ is T-invariant.

Proof. (i) $\Rightarrow$ (ii): Let $x \in X$ and $x_{0} \in X_{0}$. Then

$$
T\left(x+x_{0}\right)=x+\left(x_{0}-(x-T x)-\left(x_{0}-T x_{0}\right)\right),
$$

which shows $T\left(x+x_{0}\right) \in x+X_{0}$ since, by (i), $x-T x, x_{0}-T x_{0} \in X_{0}$. Since $x_{0} \in X_{0}$ is arbitrary, we infer $T\left(x+X_{0}\right) \subseteq x+X_{0}$.

(ii) $\Rightarrow(\mathrm{i}):$ Let $x \in X$. Then $T x=T(x+0) \in T\left(x+X_{0}\right)$, so by (ii), $T x \in x+X_{0}$, i.e., $x-T x \in X_{0}$.

The last statement follows from (i) $\Rightarrow\left(\right.$ ii) with $X_{0}:=(I-T)(X)$ and $x:=0$.

We shall also need the following well known result (see, e.g., [Ko74, Theorem 2.1]).

Lemma 2. Let $T \in L(X)$. The following statements are equivalent:

(i) $\left\|T^{n}\right\| \rightarrow 0$;

(ii) $r(T)<1$;

(iii) $\left\|T^{p}\right\|<1$ for some $p \in \mathbb{N}$;

(iv) the series $\sum_{n=0}^{\infty} T^{n}$ is uniformly convergent. 
Moreover, if one (hence each) of the above conditions holds, then $I-T$ is an isomorphism of $X$ onto $X$, and $(I-T)^{-1}=\sum_{n=0}^{\infty} T^{n}$.

The main result of this section is the following.

TheOREM 5. Let $X$ be a Banach space and $T$ be a bounded linear operator on $X$. The following statements are equivalent:

(i) $\left(T^{n}\right)_{n \in \mathbb{N}}$ is convergent in the uniform topology of $L(X)$;

(ii) there exists a linear subspace $X_{0}$ of $X$ such that

$$
(I-T)(X) \subseteq X_{0} \text { and } r\left(\left.T\right|_{X_{0}}\right)<1 ;
$$

(iii) $(I-T)(X)$ is a unique subspace satisfying (5);

(iv) $(I-T)(X)$ is closed, $X=(I-T)(X) \oplus$ Fix $T$ and $\left\|T^{n}-P\right\| \rightarrow 0$, where $P$ is the projection of $X$ onto Fix $T$ along $(I-T)(X)$;

(v) $\left\|\left.T^{p}\right|_{(I-T)(X)}\right\|<1$ for some $p \in \mathbb{N}$;

(vi) $\left\|T^{n} x-T^{n+1} x\right\| \rightarrow 0$ uniformly with respect to $x \in X$ such that $\|x-T x\| \leq 1$.

Proof. (i) $\Rightarrow\left(\right.$ ii): Set $X_{0}:=\operatorname{ker} T^{\infty}$. Since $T^{\infty} \circ T=T^{\infty}$, we infer $(I-T)(X) \subseteq X_{0}$. Clearly, $X_{0}$ is closed and $T$-invariant, and by (i), $\left(\left.T^{n}\right|_{X_{0}}\right)_{n \in \mathbb{N}}$ converges uniformly to 0 . Hence by Lemma $2, r\left(\left.T\right|_{X_{0}}\right)<1$.

(ii) $\Rightarrow$ (iii): Let $X_{0}$ be as in (ii). It suffices to show $X_{0}=(I-T)(X)$. Since, by Lemma 1, $X_{0}$ is $T$-invariant, so is $\operatorname{cl} X_{0}$ and $r\left(\left.T\right|_{\mathrm{cl} X_{0}}\right)=r\left(\left.T\right|_{X_{0}}\right)$. Hence we infer $r\left(\left.T\right|_{\mathrm{cl} X_{0}}\right)<1$, so by Lemma $2,\left.(I-T)\right|_{\mathrm{cl} X_{0}}$ is an isomorphism of $\operatorname{cl} X_{0}$ onto $\operatorname{cl} X_{0}$. Thus we get

$$
X_{0} \subseteq \operatorname{cl} X_{0}=(I-T)\left(\operatorname{cl} X_{0}\right) \subseteq(I-T)(X) \subseteq X_{0},
$$

which yields $X_{0}=(I-T)(X)$.

(iii) $\Rightarrow$ (iv): Clearly, if $X_{0}$ satisfies (5), so does $\mathrm{cl} X_{0}$. Hence by (iii), we infer $(I-T)(X)$ is closed. Set $X_{0}:=(I-T)(X)$. Claim: $X=X_{0}+$ Fix $T$. By hypothesis, $r\left(\left.T\right|_{X_{0}}\right)<1$, so by Lemma 2, there is $p \in \mathbb{N}$ such that $\left\|\left.T^{p}\right|_{X_{0}}\right\|<1$. Let $x \in X$. We shall show $\left.T^{p}\right|_{x+X_{0}}$ is a Banach contraction. Clearly, $x+X_{0}$ is closed, hence complete. Since by Lemma $1, x+X_{0}$ is $T$-invariant, it is also $T^{p}$-invariant. Now if $y_{1}, y_{2} \in x+X_{0}$, then for some $x_{1}, x_{2} \in X_{0}$, we have

$$
\begin{aligned}
\left\|T^{p} y_{1}-T^{p} y_{2}\right\| & =\left\|T^{p}\left(x+x_{1}\right)-T^{p}\left(x+x_{2}\right)\right\|=\left\|T^{p}\left(x_{1}-x_{2}\right)\right\| \\
& \leq\left\|\left.T^{p}\right|_{X_{0}}\right\|\left\|x_{1}-x_{2}\right\|=\left\|\left.T^{p}\right|_{X_{0}}\right\|\left\|y_{1}-y_{2}\right\| .
\end{aligned}
$$

Thus by the Banach Contraction Principle, Fix $\left.T^{p}\right|_{x+X_{0}}=\{z(x)\}$ for some $z(x) \in x+X_{0}$, and $T^{n p} y \rightarrow z(x)$ for all $y \in x+X_{0}$. In particular, since $x, T x, \ldots, T^{p-1} x \in x+X_{0}$, the sequences $\left(T^{n p} x\right),\left(T^{n p+1} x\right), \ldots,\left(T^{n p+p-1} x\right)$ converge to $z(x)$ as $n \rightarrow \infty$, which yields $z(x)=\lim _{n \rightarrow \infty} T^{n} x$. This means $x \in D_{T^{\infty}}$ and $z(x)=T^{\infty} x$; moreover, $T^{\infty} x \in x+X_{0}$, i.e., $x-T^{\infty} x \in X_{0}$. Thus the equality $x=\left(x-T^{\infty} x\right)+T^{\infty} x$ shows $x \in X_{0}+\operatorname{Fix} T$, which proves 
our claim. Furthermore, the above argument yields Fix $T \cap\left(x+X_{0}\right)=\left\{T^{\infty} x\right\}$ for $x \in X$; in particular, Fix $T \cap X_{0}=\{0\}$. So we infer $X=X_{0} \oplus \operatorname{Fix} T$.

Now we shall show $\left\|T^{n}-P\right\| \rightarrow 0$. We have proved that $P=T^{\infty}$, so given $n \in \mathbb{N}, P=T^{n} \circ P$. Hence, for $x \in X$,

$$
\left\|T^{n} x-P x\right\|=\left\|T^{n}(x-P x)\right\| \leq\left\|\left.T^{n}\right|_{X_{0}}\right\|\|I-P\|\|x\|,
$$

so $\left\|T^{n}-P\right\| \leq\left\|\left.T^{n}\right|_{X_{0}}\right\|\|I-P\|$. By hypothesis and Lemma $2,\left\|\left.T^{n}\right|_{X_{0}}\right\| \rightarrow 0$, so we infer $\left(T^{n}\right)_{n \in \mathbb{N}}$ uniformly converges to $P$.

$(\mathrm{iv}) \Rightarrow(\mathrm{v})$ : Let $P$ be the projection as in (iv). Then $\left.\left(T^{n}-P\right)\right|_{(I-T)(X)}=$ $\left.T^{n}\right|_{(I-T)(X)}$, so by (iv), $\left\|\left.T^{n}\right|_{(I-T)(X)}\right\| \rightarrow 0$. By Lemma 2 , (v) holds.

$(\mathrm{v}) \Rightarrow(\mathrm{vi})$ : By Lemma 2, (v) implies that $\left\|\left.T^{n}\right|_{(I-T)(X)}\right\| \rightarrow 0$, which yields (vi).

Finally, it is obvious that (iv) implies (i), so conditions (i)-(iv) are all equivalent. Thus, to complete the proof, it suffices to show (vi) $\Rightarrow$ (ii). By Lemma 2, we easily infer that (vi) implies $r\left(\left.T\right|_{(I-T)(X)}\right)<1$, so (ii) holds with $X_{0}:=(I-T)(X)$.

As an immediate consequence of Theorem 5 , we get the following.

Corollary 1. Let $X$ be a Banach space and $T \in L(X)$. If there exists a linear subspace $X_{0}$ of $X$ such that $(I-T)(X) \subseteq X_{0}$ and $\left\|\left.T^{p}\right|_{X_{0}}\right\|<1$ for some $p \in \mathbb{N}$, then $X_{0}=(I-T)(X), X=X_{0} \oplus$ Fix $T$ and $\left(T^{n}\right)_{n \in \mathbb{N}}$ uniformly converges to the projection of $X$ onto Fix $T$ along $X_{0}$. Hence, for any $x \in X$,

$$
\left\{\lim _{n \rightarrow \infty} T^{n} x\right\}=\operatorname{Fix} T \cap\left(x+X_{0}\right) .
$$

Proof. By Lemma $2, r\left(\left.T\right|_{X_{0}}\right)<1$. Hence by Theorem $5(($ iii $) \Rightarrow($ iv $))$, we infer $X_{0}=(I-T)(X)$, which together with (iii) $\Rightarrow(\mathrm{v})$ shows that $X=$ $X_{0} \oplus$ Fix $T$ and $\left(T^{n}\right)_{n \in \mathbb{N}}$ uniformly converges to the projection of $X$ onto Fix $T$ along $X_{0}$. Hence, given $x \in X, x=x_{0}+T^{\infty} x$ for some $x_{0} \in X_{0}$, which implies $T^{\infty} x \in \operatorname{Fix} T \cap\left(x+X_{0}\right)$. Since Fix $T \cap\left(x+X_{0}\right)$ is a singleton, we get (4).

3. Limits of iterates of some positive linear operators. In this section we present an application of Corollary 1 to the study of asymptotic behaviour of iterates of linear operators on the Banach space $C(\Omega)$, where $\Omega$ is a compact topological space. The following theorem deals with a class of some positive linear operators. (Recall that $T \in L(C(\Omega)$ ) is positive if $T f \geq 0$ whenever $f \geq 0$.) In particular, we shall show that this class contains multivariate Bernstein operators (with $\Omega$ being the standard $N$-simplex or the $N$-cube) as well as some generalized Bernstein operators studied by many authors. We denote by $\delta_{k l}$ Kronecker's delta symbol.

TheOREM 6. Let $\Omega$ be a compact Hausdorff topological space, $m \in \mathbb{N}$ and $a_{0}, a_{1}, \ldots, a_{m} \in \Omega$ be pairwise distinct. Let $\varphi_{0}, \varphi_{1}, \ldots, \varphi_{m} \in C(\Omega)$ be 
nonnegative and such that $\sum_{k=0}^{m} \varphi_{k}=1$. Set

$$
T f:=\sum_{k=0}^{m} f\left(a_{k}\right) \varphi_{k} \quad \text { for } f \in C(\Omega) .
$$

Assume that the set $I:=\left\{i \in\{0,1, \ldots, m\}:\left\|\varphi_{i}\right\|=1\right\}$ is nonempty, and $I=\left\{i_{1}, \ldots, i_{p}\right\}$, where $i_{1}<\cdots<i_{p}$. If

$$
\varphi_{i_{k}}\left(a_{i_{k}}\right)=1 \quad \text { for } k=1, \ldots, p \quad \text { and } \quad \bigcap_{k=1}^{p} \varphi_{i_{k}}^{-1}(0)=\emptyset,
$$

then $\operatorname{dim} \operatorname{Fix} T=p$, and there exists a basis $\left\{\psi_{1}, \ldots, \psi_{p}\right\}$ for Fix $T$ such that $\psi_{k}\left(a_{i_{l}}\right)=\delta_{k l}$ for $k, l \in\{1, \ldots, p\}$. Moreover, the sequence $\left(T^{n}\right)_{n \in \mathbb{N}}$ uniformly converges to the operator $T^{\infty}$ given by the following formula:

$$
T^{\infty} f=\sum_{k=1}^{p} f\left(a_{i_{k}}\right) \psi_{k} \quad \text { for } f \in C(\Omega) .
$$

Proof. Set $X:=C(\Omega)$ and $X_{0}:=\left\{f \in X: f\left(a_{i_{k}}\right)=0\right.$ for $\left.k=1, \ldots, p\right\}$. Clearly, codim $X_{0}=p$, because $\Omega$ is a normal space. Observe that we have $(I-T)(X) \subseteq X_{0}$. Indeed, let $f \in X$ and $k \in\{1, \ldots, p\}$. Then

$$
((I-T) f)\left(a_{i_{k}}\right)=\sum_{j=0}^{p}\left(f\left(a_{i_{k}}\right)-f\left(a_{j}\right)\right) \varphi_{j}\left(a_{i_{k}}\right) .
$$

Since $\varphi_{i_{k}}\left(a_{i_{k}}\right)=1$ and $\sum_{j=0}^{p} \varphi_{j}=1$, we get $\varphi_{j}\left(a_{i_{k}}\right)=0$ for $j \neq i_{k}$. Thus $((I-T) f)\left(a_{i_{k}}\right)=0$, which means $(I-T) f \in X_{0}$.

We shall show $\left\|\left.T\right|_{X_{0}}\right\|<1$. If $p=m+1$, then $\left.T\right|_{X_{0}}=0$. So let $p \leq m$ and $f \in X_{0}$. Then

$$
\begin{aligned}
|(T f)(t)| & \leq \sum_{j \in\{0, \ldots, N\} \backslash\left\{i_{1}, \ldots, i_{p}\right\}}\left|f\left(a_{j}\right)\right| \varphi_{j}(t) \leq\|f\|\left(1-\sum_{k=1}^{p} \varphi_{i_{k}}(t)\right) \\
& \leq\|f\|\left(1-\sum_{k=1}^{p} \varphi_{i_{k}}\left(t_{0}\right)\right)
\end{aligned}
$$

for some $t_{0} \in \Omega$ since the function $t \mapsto 1-\sum_{k=1}^{p} \varphi_{i_{k}}(t)$ attains its maximum on $\Omega$. Set $\alpha:=1-\sum_{k=1}^{p} \varphi_{i_{k}}\left(t_{0}\right)$. Clearly, $\alpha \geq 0$, and $\alpha<1$ since $\bigcap_{i \in I} \varphi_{i}^{-1}(0)=\emptyset$. Hence $\left.T\right|_{X_{0}}$ is an $\alpha$-contraction.

Thus by Corollary $1,\left(T^{n}\right)_{n \in \mathbb{N}}$ uniformly converges, $\lim _{n \rightarrow \infty} T^{n} f \in f+X_{0}$ for $f \in X$, and $X=X_{0} \oplus \operatorname{Fix} T$. Since $\operatorname{codim} X_{0}=p$, we get $\operatorname{dim} \operatorname{Fix} T=p$. Since $\Omega$ is normal, by the Urysohn Lemma there exist $g_{1}, \ldots, g_{p} \in X$ such that $g_{k}\left(a_{i_{l}}\right)=\delta_{k l}$ for $k, l \in\{1, \ldots, p\}$. Set

$$
\psi_{k}:=T^{\infty} g_{k} \quad \text { for } k=1, \ldots, p .
$$


Clearly, $\psi_{1}, \ldots, \psi_{p} \in$ Fix $T$, and since $\psi_{k} \in g_{k}+X_{0}, \psi_{k}\left(a_{i_{l}}\right)=g_{k}\left(a_{i_{l}}\right)=$ $\delta_{k l}$, so we infer $\psi_{1}, \ldots, \psi_{p}$ are linearly independent. Thus $\left\{\psi_{1}, \ldots, \psi_{p}\right\}$ is a basis for Fix $T$ since $\operatorname{dim} \operatorname{Fix} T=p$. Now if $f \in X$, then, by (4), $\left\{T^{\infty} f\right\}=$ Fix $T \cap\left(f+X_{0}\right)$. On the other hand, it is easily seen that $\sum_{k=1}^{p} f\left(a_{i_{k}}\right) \psi_{k} \in$ Fix $T \cap\left(f+X_{0}\right)$, which yields $(6)$.

REMARK 1. Observe that under the assumptions of Theorem 6 , if $J$ is a nonempty subset of $I$ such that $\bigcap_{j \in J} \varphi_{j}^{-1}(0)=\emptyset$, then $J=I$. Indeed, suppose that, on the contrary, there exists $i \in I \backslash J$. Then $\varphi_{i}(a)=1$ for some $a \in \Omega$, so $\sum_{k \neq i} \varphi_{k}(a)=0$; in particular, $\varphi_{j}(a)=0$ for each $j \in J$, which yields a contradiction.

REMARK 2. As observed by the referee, it is possible to give another proof of Theorem 6. Namely, it is easy to see that the uniform covergence of $\left(T^{n}\right)_{n \in \mathbb{N}}$ is equivalent to the covergence of the powers of the following matrix:

$$
P:=\left(\varphi_{j}\left(a_{i}\right)\right)_{i, j=0}^{m},
$$

which is a stochastic (in rows) matrix. Necessary and sufficient conditions for the convergence of $\left(P^{n}\right)_{n \in \mathbb{N}}$ are well known (see, e.g., [Ga59, Chapter 13]) and can be applied here. Actually, such an approach was also used in [KR67, OT02, We97], but in those papers it led to arduous calculations. Our method is more abstract and it seems to be simpler.

Corollary 2. Let $a_{0}, a_{1}, \ldots, a_{m} \in[0,1]$ be such that $0=a_{0}<a_{1}<$ $\cdots<a_{m}=1$. Assume that $\varphi_{0}, \varphi_{1}, \ldots \varphi_{m} \in C[0,1]$ are nonnegative and such that

$$
\sum_{k=0}^{m} \varphi_{k}=1, \quad \varphi_{0}(0)=\varphi_{m}(1)=1 \quad \text { and } \quad \varphi_{0}^{-1}(0) \cap \varphi_{m}^{-1}(0)=\emptyset .
$$

Let $T$ be defined by (5). Then there is a unique function $\psi \in \operatorname{Fix} T$ such that $\psi(0)=0$ and $\psi(1)=1$; moreover, $\left(T^{n}\right)_{n \in \mathbb{N}}$ uniformly converges to the operator $T^{\infty}$ given by the following formula:

$$
\left(T^{\infty} f\right)(t)=f(0)+(f(1)-f(0)) \psi(t) \quad \text { for } f \in C[0,1] \text { and } t \in[0,1] .
$$

In particular, if $T e_{1}=e_{1}$ where $e_{1}:=\mathrm{id}_{[0,1]}$, then

$$
\left(T^{\infty} f\right)(t)=f(0)+(f(1)-f(0)) t .
$$

Proof. Let $I$ be the set as in Theorem 6. By hypothesis and Remark 1, $I=\{0, m\}$. Since $\varphi_{0}\left(a_{0}\right)=\varphi_{m}\left(a_{m}\right)=1$, we infer in view of Theorem 6 that $\operatorname{dim} \operatorname{Fix} T=2$, and there is a basis $\left\{\psi_{1}, \psi_{2}\right\}$ for Fix $T$ such that $\psi_{1}(0)=$ $\psi_{2}(1)=1$ and $\psi_{1}(1)=\psi_{2}(0)=0$. Let $e_{0}(t):=1$ for $t \in[0,1]$. Then $e_{0} \in$ Fix $T$ since $T e_{0}=\sum_{k=0}^{m} \varphi_{k}=1$. Moreover, $\left\{e_{0}, \psi_{2}\right\}$ is linearly independent, so Fix $T=\operatorname{span}\left(e_{0}, \psi_{2}\right)$ since $\operatorname{dim} \operatorname{Fix} T=2$. Hence $\psi_{1} \in \operatorname{span}\left(e_{0}, \psi_{2}\right)$, from which we infer $\psi_{1}=e_{0}-\psi_{2}$. Set $\psi:=\psi_{2}$. Then $\psi$ has all the properties we 
need, and by (6), we obtain $T^{\infty} f=f(0)\left(e_{0}-\psi\right)+f(1) \psi$, i.e., (7) holds. Finally, if $\eta \in \operatorname{Fix} T$ is such that $\eta(0)=0$ and $\eta(1)=1$, then $\eta=T^{\infty} \eta=$ $\eta(0)+(\eta(1)-\eta(0)) \psi=\psi$, which shows the uniqueness of $\psi$.

In what follows we discuss some consequences of Theorem 6 . We start with a proof of the Kelisky-Rivlin theorem via Corollary 2.

Proof of Theorem 1. For $k=0,1, \ldots, m$ and $t \in[0,1]$, set

$$
a_{k}:=\frac{k}{m} \quad \text { and } \quad \varphi_{k}(t):=\left(\begin{array}{c}
m \\
k
\end{array}\right) t^{k}(1-t)^{m-k} .
$$

In particular, $\varphi_{0}(t)=(1-t)^{m}$ and $\varphi_{m}(t)=t^{m}$, so it is clear that the assumptions of Corollary 2 are satisfied. Moreover, since $B_{m} e_{1}=e_{1}$, the last statement of Corollary 2 yields (2).

Corollary 2 also generalizes [AR03, Theorem 4] in which the authors use the assumptions $\sum_{k=0}^{m} a_{k} \varphi_{k}(t)=t$ (this means $\left.T e_{1}=e_{1}\right)$, and $\min \left\{\varphi_{0}(t)+\right.$ $\left.\varphi_{m}(t): t \in[0,1]\right\}>0$, which is equivalent to $\varphi_{0}^{-1}(0) \cap \varphi_{m}^{-1}(0)=\emptyset$.

Now we turn our attention to the so-called $q$-Bernstein operators $B_{m, q}$ $(q \in(0, \infty))$ introduced by Phillips [Ph97]. For $f \in C[0,1]$ and $t \in[0,1]$, we set

$$
\left(B_{m, q} f\right)(t):=\sum_{k=0}^{m} f\left(\frac{[k]_{q}}{[m]_{q}}\right)\left[\begin{array}{c}
m \\
k
\end{array}\right]_{q} t^{k} \prod_{s=0}^{m-k-1}\left(1-q^{s} t\right),
$$

where $[k]_{q}:=1+q+\cdots+q^{k-1}$ for $k>0,[0]_{q}:=0$ and $\left[\begin{array}{c}m \\ k\end{array}\right]_{q}$ is the $q$-binomial coefficient defined by

$$
\left[\begin{array}{c}
m \\
k
\end{array}\right]_{q}:=\frac{[m]_{q}[m-1]_{q} \ldots[m-(k-1)]_{q}}{[1]_{q}[2]_{q} \ldots[k]_{q}} \quad \text { if } 1 \leq k \leq m,
$$

and $\left[\begin{array}{c}m \\ 0\end{array}\right]_{q}:=1$. Moreover, $\prod_{s=0}^{-1}(\cdots)$ denotes 1 . Clearly, $[k]_{1}=k$ and $\left[\begin{array}{c}m \\ k\end{array}\right]_{1}=$ $\left(\begin{array}{c}m \\ k\end{array}\right)$, so the classical Bernstein operator coincides with $B_{m, q}$ when $q=1$. It turns out that the Kelisky-Rivlin theorem can be extended to $q$-Bernstein operators according to [OT02, Theorem 3.1].

TheOREM 7 (Oruç-Tuncer). Let $q$ be a positive real number, $m \in \mathbb{N}$, $f \in C[0,1]$ and $t \in[0,1]$. Then the sequence $\left(\left(B_{m, q}^{n} f\right)(t)\right)_{n \in \mathbb{N}}$ converges and

$$
\lim _{n \rightarrow \infty}\left(B_{m, q}^{n} f\right)(t)=f(0)+(f(1)-f(0)) t .
$$

Observe that for $q \in(0,1]$, the conclusion of Theorem 7 can easily be derived from Corollary 2. Indeed, set

$$
a_{k}:=\frac{[k]_{q}}{[m]_{q}} \quad \text { and } \quad \varphi_{k}(t):=\left[\begin{array}{c}
m \\
k
\end{array}\right]_{q} t^{k} \prod_{s=0}^{m-k-1}\left(1-q^{s} t\right)
$$


for $k=0, \ldots, m$ and $t \in[0,1]$. Then $B_{m, q} f=\sum_{k=0}^{m} f\left(a_{k}\right) \varphi_{k}$. Since $q \in(0,1]$, all $\varphi_{k}$ are nonnegative. It was shown in [Ph97] that $B_{m, q} e_{i}=e_{i}$ for $i=0,1$. Thus $B_{m, q} e_{0}=e_{0}$ yields $\sum_{k=0}^{m} \varphi_{k}=1$. Since

$$
\varphi_{0}(t)=(1-t)(1-q t) \ldots\left(1-q^{m-1} t\right) \text { and } \varphi_{m}(t)=t^{m},
$$

we infer that all the assumptions of Corollary 2 are satisfied, so (8) holds. Moreover, the sequence $\left(B_{m, q}^{n}\right)_{n \in \mathbb{N}}$ is uniformly convergent.

Now we show that Corollary 2 yields yet another generalization of the Kelisky-Rivlin theorem given by Gonska and Piţul [GP05, Theorem 3.2]. For $m \in \mathbb{N}$, we consider the following operator $V_{m}$ on $C[0,1]$ defined by King [Ki03]:

$$
\left(V_{m} f\right)(t):=\sum_{k=0}^{m} f\left(\frac{k}{m}\right)\left(\begin{array}{c}
m \\
k
\end{array}\right)\left(r_{m}(t)\right)^{k}\left(1-r_{m}(t)\right)^{m-k},
$$

where $r_{m}:[0,1] \rightarrow[0,1]$ is a continuous function. Clearly, $V_{m}=B_{m}$ when $r_{m}=\mathrm{id}_{[0,1]}$.

Theorem 8 (Gonska-Piţul). Given $m \in \mathbb{N}$, define the function $r_{m}^{*}$ by $r_{m}^{*}(t):= \begin{cases}t^{2} & \text { if } m=1 \\ 1-1 /(2 m-2)+\left[(m /(m-1)) t^{2}+1 /(2 m-2)^{2}\right]^{1 / 2} & \text { if } m \geq 2 .\end{cases}$

Denote by $V_{m, *}$ the corresponding operator $V_{m}$. Then, for any $f \in C[0,1]$ and $t \in[0,1]$, the sequence $\left(\left(V_{m, *}^{n} f\right)(t)\right)_{n \in \mathbb{N}}$ converges and

$$
\lim _{n \rightarrow \infty}\left(V_{m, *}^{n} f\right)(t)=f(0)+(f(1)-f(0)) t^{2} .
$$

Observe that $r_{m}^{*}(0)=0$ and $r_{m}^{*}(1)=1$. Now, with the help of Corollary 2, we may easily generalize Theorem 8 in the following way.

Theorem 9. Given $m \in \mathbb{N}$, assume that a function $r_{m}$ is such that $r_{m}(0)=0$ and $r_{m}(1)=1$. Then the sequence $\left(V_{m}^{n}\right)_{n \in \mathbb{N}}$ uniformly converges, and there exists a unique function $\psi \in$ Fix $V_{m}$ such that $\psi(0)=0$ and $\psi(1)=1$. Moreover, for any $f \in C[0,1]$ and $t \in[0,1]$,

$$
\lim _{n \rightarrow \infty}\left(V_{m}^{n} f\right)(t)=f(0)+(f(1)-f(0)) \psi(t) .
$$

In particular, $\psi(t)=t^{2}$ if $r_{m}=r_{m}^{*}$.

Proof. Set $a_{k}:=k / m$ and $\varphi_{k}(t):=\left(\begin{array}{c}m \\ k\end{array}\right)\left(r_{m}(t)\right)^{k}\left(1-r_{m}(t)\right)^{m-k}$ for $k=0, \ldots, m$. Clearly, all $\varphi_{k}$ are nonnegative and $\sum_{k=0}^{m} \varphi_{k}=1$. Since $\varphi_{0}(t)=\left(1-r_{m}(t)\right)^{m}$ and $\varphi_{m}(t)=\left(r_{m}(t)\right)^{m}$, in view of the hypothesis $\varphi_{0}$ and $\varphi_{m}$ satisfy the assumptions of Corollary 2 . Hence $\left(V_{m}^{n}\right)_{n \in \mathbb{N}}$ is uniformly convergent and (10) holds. Moreover, if $r_{m}=r_{m}^{*}$, then $e_{2} \in$ Fix $V_{m}$, where $e_{2}(t):=t^{2}$ (see [Ki03]). Since $e_{2}(0)=0$ and $e_{2}(1)=1$, Corollary 2 yields $\psi=e_{2}$. 
Now we consider the bivariate Bernstein polynomials over a triangle, which were introduced by Lorentz [Lo53]. Let $\triangle$ be the standard triangle in $\mathbb{R}^{2}$, i.e.,

$$
\triangle:=\{(s, t): s, t \geq 0 \text { and } s+t \leq 1\} .
$$

For $m \in \mathbb{N}, f \in C(\triangle)$ and $(s, t) \in \triangle$, define

$$
B_{m, \triangle} f:=\sum_{i=0}^{m} \sum_{j=0}^{m-i} f(i / m, j / m) \varphi_{i j},
$$

where $\varphi_{i j}(s, t):=m ! /(i ! j !(m-i-j) !) s^{i} t^{j}(1-s-t)^{m-i-j}$. The following result was proved by Chang and Feng [CF86] (cf. also [CF93], [AA96], [We97]).

TheOREM 10. For any $m \in \mathbb{N}$, the sequence $\left(B_{m, \triangle}^{n}\right)_{n \in \mathbb{N}}$ strongly converges to the operator $B_{\triangle}^{\infty}$ (independent of $m$ ) given by the following formula for $f \in C(\triangle)$ and $(s, t) \in \triangle$ :

$$
\left(B_{\triangle}^{\infty} f\right)(s, t)=f(0,0)+(f(1,0)-f(0,0)) s+(f(0,1)-f(0,0)) t .
$$

As already mentioned, all known proofs of Theorem 10 require rather hard calculations. Now we shall show that Theorem 10 can easily be derived from Theorem 6 . In particular, the sequence $\left(B_{m, \triangle}^{n}\right)_{n \in \mathbb{N}}$ uniformly converges to $B_{\triangle}^{\infty}$, which improves Theorem 10 . Clearly, $B_{m, \triangle}$ can be put down in the form (5), but it will be more convenient to work with double indices, so we shall identify elements of $I$ defined in Theorem 6 with some pairs $(i, j)$.

Proof of Theorem 10. Set $a_{i, j}:=(i / m, j / m)$ for $i=0, \ldots, m$ and $j=$ $0, \ldots, m-i$. It is well known that

$$
B_{m, \triangle} f_{i}=f_{i} \quad \text { for } i=1,2,3,
$$

where $f_{1}(s, t):=1, f_{2}(s, t):=s$ and $f_{3}(s, t):=t$ (cf. [Lo53]). Hence $B_{m, \triangle} f_{1}$ $=f_{1}$ yields $\sum_{i=0}^{m} \sum_{j=0}^{m-i} \varphi_{i j}=1$. Since $\varphi_{00}(s, t)=(1-s-t)^{m}, \varphi_{m 0}(s, t)=s^{m}$ and $\varphi_{0 m}(s, t)=t^{m}$, we infer $(0,0),(m, 0),(0, m) \in I$ and $\varphi_{00}^{-1}(0) \cap \varphi_{m 0}^{-1}(0) \cap$ $\varphi_{0 m}^{-1}(0)=\emptyset$. Consequently, by Remark 1 ,

$$
I=\{(0,0),(m, 0),(0, m)\} .
$$

Moreover, $\varphi_{00}\left(a_{00}\right)=\varphi_{m 0}\left(a_{m 0}\right)=\varphi_{0 m}\left(a_{0 m}\right)=1$, so by Theorem 6 , Fix $B_{m, \triangle}$ is 3-dimensional, and there is a basis $\left\{\psi_{1}, \psi_{2}, \psi_{3}\right\}$ for Fix $B_{m, \Delta}$ (which coincides with $\operatorname{span}\left(f_{1}, f_{2}, f_{3}\right)$ in view of $\left.(12)\right)$ such that $\psi_{1}(0,0)=$ $\psi_{2}(1,0)=\psi_{3}(0,1)=1$, and each of these functions vanishes at the remaining vertices of $\triangle$. Hence we easily find that $\psi_{1}(s, t)=1-s-t, \psi_{2}(s, t)=s$ and $\psi_{3}(s, t)=t$ for $(s, t) \in \triangle$, so (11) follows immediately from (6).

REMARK 3. The above argument can easily be adapted to the case of the Bernstein operator $B_{m, S}$, where $S$ is the standard $N$-simplex. In this case $\operatorname{dim}$ Fix $B_{m, S}=N+1$ and given $f \in C(S), B_{S}^{\infty} f$ is the linear function interpolating $f$ at each vertex of $S$. 
Finally, we consider the tensor product Bernstein operators (cf. [Lo53, p. 51]). Recall that the bivariate tensor product Bernstein operator $B_{l} \otimes B_{m}$ is defined on $C\left([0,1]^{2}\right)$ by

$$
\left(\left(B_{l} \otimes B_{m}\right) f\right)(s, t):=\sum_{i=0}^{l} \sum_{j=0}^{m} f(i / l, j / m)\left(\begin{array}{c}
l \\
i
\end{array}\right)\left(\begin{array}{c}
m \\
j
\end{array}\right) s^{i}(1-s)^{l-i} t^{j}(1-t)^{m-j} .
$$

It seems that the asymptotic behaviour of the iterates of $B_{l} \otimes B_{m}$ has not been studied yet. Now we give a formula for the limit of the iterates.

Theorem 11. For any $l, m \in \mathbb{N}$, the sequence $\left(\left(B_{l} \otimes B_{m}\right)^{n}\right)_{n \in \mathbb{N}}$ uniformly converges to the operator $L$ (independent of $l$ and $m$ ) given by the following formula for $f \in C\left([0,1]^{2}\right)$ and $s, t \in[0,1]$ :

$$
\begin{aligned}
(L f)(s, t)= & f(0,0)+(f(1,0)-f(0,0)) s+(f(0,1)-f(0,0)) t \\
& +(f(0,0)+f(1,1)-f(1,0)-f(0,1)) s t .
\end{aligned}
$$

Proof. For $i=0,1, \ldots, l$ and $j=0,1, \ldots, m$, set $a_{i j}:=(i / l, j / m)$, and

$$
\varphi_{i j}(s, t):=\left(\begin{array}{c}
l \\
i
\end{array}\right)\left(\begin{array}{c}
m \\
j
\end{array}\right) s^{i}(1-s)^{l-i} t^{j}(1-t)^{m-j} .
$$

Then $\sum_{i=0}^{l} \sum_{j=0}^{m} \varphi_{i j}=1$ and $\left(B_{l} \otimes B_{m}\right) f=\sum_{i=0}^{l} \sum_{j=0}^{m} f\left(a_{i j}\right) \varphi_{i j}$. Consider the following four functions:

$$
\begin{aligned}
\varphi_{00}(s, t) & =(1-s)^{l}(1-t)^{m}, & \varphi_{l 0}(s, t) & =s^{l}(1-t)^{m}, \\
\varphi_{l m}(s, t) & =s^{l} t^{m}, & \varphi_{0 m}(s, t) & =(1-s)^{l} t^{m} .
\end{aligned}
$$

Then $\varphi_{00}\left(a_{00}\right)=\varphi_{l 0}\left(a_{l 0}\right)=\varphi_{l m}\left(a_{l m}\right)=\varphi_{0 m}\left(a_{0 m}\right)=1$ and the four functions have no common zero. By Remark $1, I=\{(0,0),(l, 0),(l, m),(0, m)\}$, so Theorem 6 yields $\operatorname{dim}$ Fix $B_{l} \otimes B_{m}=4$. For $s, t \in[0,1]$, set

$$
f_{1}(s, t):=1, f_{2}(s, t):=s, f_{3}(s, t):=t, f_{4}(s, t):=s t .
$$

Then $\left\{f_{1}, f_{2}, f_{3}, f_{4}\right\}$ is a basis for Fix $B_{l} \otimes B_{m}$. For $i=1,2,3,4$, let $\psi_{i}$ be the function as in Theorem 6 . Then $\psi_{i} \in \operatorname{span}\left(f_{1}, f_{2}, f_{3}, f_{4}\right)$ and hence, after simple calculations, we get

$$
\begin{array}{ll}
\psi_{1}(s, t)=1-s-t+s t, & \psi_{2}(s, t)=s-s t \\
\psi_{3}(s, t)=s t, & \psi_{4}(s, t)=t-s t .
\end{array}
$$

Now (13) follows directly from (6).

The above argument can easily be carried over to the case of the tensor product Bernstein operators on $C\left([0,1]^{N}\right)$ for any $N \in \mathbb{N}$. Then, as the reader may verify, we get the following formula for the limit $L$ of iterates of this operator:

$$
(L f)\left(t_{1}, \ldots, t_{N}\right)=\sum_{\left(\varepsilon_{1}, \ldots, \varepsilon_{N}\right) \in V} f\left(\varepsilon_{1}, \ldots, \varepsilon_{N}\right) p_{\varepsilon_{1}}\left(t_{1}\right) \ldots p_{\varepsilon_{N}}\left(t_{N}\right),
$$


where $f \in C\left([0,1]^{N}\right), t_{1}, \ldots, t_{N} \in[0,1], V:=\{0,1\}^{\{1, \ldots, N\}}$, and for $s \in[0,1]$, $p_{0}(s):=1-s$ and $p_{1}(s):=s$.

Acknowledgements. The author is grateful to the referee and to Professor Tadeusz Figiel for some useful suggestions.

\section{References}

[AA96] U. Abel and G. Arends, The powers of the bivariate Bernstein operators, Extracta Math. 11 (1996), 389-404.

[AR03] O. Agratini and I. A. Rus, Iterates of a class of discrete linear operators via contraction principle, Comment. Math. Univ. Carolin. 44 (2003), 555-563.

[CF86] G. Z. Chang and Y. Y. Feng, Limit of iterates for Bernstein polynomials defined on higher-dimensional domains, Kexue Tongbao (English Ed.) 31 (1986), 157160.

[CF93] F. L. Chen and Y. Y. Feng, Limit of iterates for Bernstein polynomials defined on a triangle, Appl. Math. J. Chinese Univ. Ser. B 8 (1993), 45-53.

[DS57] N. Dunford and J. T. Schwartz, Linear Operators. I. General Theory, Pure Appl. Math. 7, Interscience, London, 1958.

[Em07] E. Yu. Emel'yanov, Non-spectral Asymptotic Analysis of One-Parameter Operator Semigroups, Oper. Theory Adv. Appl. 173, Birkhäuser, Basel, 2007.

[Ga59] F. R. Gantmacher, The Theory of Matrices, Chelsea, New York, 1959.

[Ge86] Y. Ge, Review of [Li86], Zbl 0601.60084.

[GP05] H. Gonska and P. Piţul, Remarks on an article of J. P. King, Comment. Math. Univ. Carolin. 46 (2005), 645-652.

[Ja07] J. Jachymski, The contraction principle for mappings on a metric space with a graph, Proc. Amer. Math. Soc. 136 (2008), 1359-1373.

[KR67] R. P. Kelisky and T. J. Rivlin, Iterates of Bernstein polynomials, Pacific J. Math. 21 (1967), 511-520.

[Ki03] J. P. King, Positive linear operators which preserve $x^{2}$, Acta Math. Hungar. 99 (2003), 203-208.

[Ko73] J. J. Koliha, Convergent and stable operators and their generalization, J. Math. Anal. Appl. 43 (1973), 778-794.

[Ko74] -, Power convergence and pseudoinverses of operators in Banach spaces, J. Math. Anal. Appl. 48 (1974), 446-469.

[Li86] H. Li, Equivalent conditions for the convergence of a sequence $\left\{B^{n}\right\}_{n=1}^{\infty}$, Acta Math. Sinica 29 (1986), 285-288 (in Chinese).

[Li74] M. Lin, On the uniform ergodic theorem, Proc. Amer. Math. Soc. 43 (1974), 337-340.

[Lo53] G. G. Lorentz, Bernstein Polynomials, Math. Exp. 8, Univ. of Toronto Press, Toronto, 1953.

[LZ94] Yu. Lyubich and J. Zemánek, Precompactness in the uniform ergodic theory, Studia Math. 112 (1994), 89-97.

[MZ93] M. Mbekhta et J. Zemánek, Sur le théorème ergodique uniforme et le spectre, C. R. Acad. Sci. Paris Sér. I Math. 317 (1993), 1155-1158.

[OT02] H. Oruç and N. Tuncer, On the convergence and iterates of q-Bernstein polynomials, J. Approx. Theory 117 (2002), 301-313.

[Ph97] G. M. Phillips, Bernstein polynomials based on the q-integers, Ann. Numer. Math. 4 (1997), 511-518. 
[Rä93] F. Räbiger, Stability and ergodicity of dominated semigroups. I. The uniform case, Math. Z. 214 (1993), 43-53.

[Ru04] I. A. Rus, Iterates of Bernstein operators, via contraction principle, J. Math. Anal. Appl. 292 (2004), 259-261.

[We97] H.-J. Wenz, On the limits of (linear combinations of) iterates of linear operators, J. Approx. Theory 89 (1997), 219-237.

Institute of Mathematics

Technical University of Łódź

Wólczańska 215

93-005 Łódź, Poland

E-mail: jachym@p.lodz.pl 